\title{
HISTORIA
}

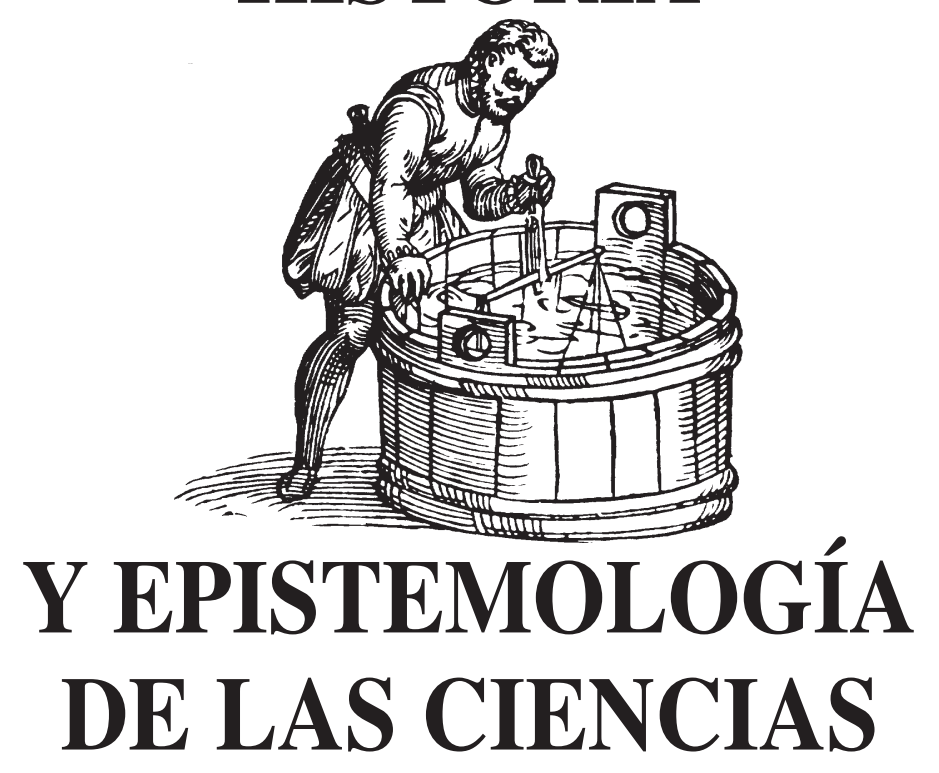

\section{ENSEÑANZA DE LAS CIENCIAS Y FILOSOFÍA*}

PARUELO, JORGE

Universidad de Buenos Aires

Lautaro 827. Dto B. 1406 Ciudad de Buenos Aires. Argentina

jparuelo@mail.retina.ar

Resumen. La epistemología es una de las herramientas necesarias para el desarrollo de nuevas estrategias para la enseñanza de las ciencias. Sin embargo, no está claro el rol que esta disciplina juega en este proceso. En este trabajo presento una serie de ejemplos extraídos de manuales de uso habitual en la enseñanza de las ciencias donde se detecta la falta de un correcto análisis epistemológico. Identifico dos roles para los filósofos de la ciencia: la tarea de formación epistemológica de los docentes de ciencia y la investigación acerca de los costos epistemológicos de la transposición didáctica y de los supuestos que tiene la ciencia enseñada.

Palabras clave. Epistemología, transposición, operacionalismo, causalidad.

Summary. Epistemology is a valuable tool for the design of new strategies in science teaching. However, the role the discipline plays in this process is not generally clear. In this paper I present several examples taken from standard science books that show lack of a correct epistemological analysis. I identify two roles for philosophers of science: the task of epistemological training of science teachers and the research of the epistemological costs of didactic transposition and of the implicit assumptions of the theory being taught.

Keywords. Epistemology, transposition, operationalism, causality 


\section{INTRODUCCIÓN}

No hay duda de que el interés por mejorar la enseñanza de las ciencias ha crecido en los últimos tiempos tanto en los países de bajo desarrollo científico tecnológico como en aquéllos más avanzados en la materia. Tradicionalmente era una preocupación de los profesores y los especialistas en educación, pero la inquietud se ha trasladado ahora a aquellos investigadores que hasta aquí se dedicaban a sus respectivas disciplinas científicas. Este creciente interés genera un incremento en la cantidad, calidad y especificidad en las investigaciones en el tema. Se incrementa así la búsqueda de nuevas estrategias para abordar la enseñanza de las ciencias, incluso en los primeros niveles de la educación formal. Junto con esto crece la cantidad de grupos de investigación especializados en la enseñanza de las ciencias.

La epistemología surge naturalmente como una herramienta útil y necesaria para el desarrollo de nuevas estrategias. Esto ha sido puesto en evidencia en varios trabajos desarrollados sobre todo en los últimos quince años ${ }^{1}$. Es habitual que investigadores en enseñanza de las ciencias aborden temas vinculados a la epistemología. No es tan habitual, sin embargo, que los filósofos de la ciencia aborden el tema ${ }^{2}$. Es raro encontrar trabajos sobre la relación entre filosofía de la ciencia y enseñanza en los congresos de filosofía ${ }^{3}$.

Existe una dificultad inicial respecto del significado que se da al término epistemología entre los investigadores en enseñanza de las ciencias. En algunos casos se considera este término con un significado ligado a teoría del conocimiento y en tal sentido asociado con determinadas posturas respecto del aprendizaje. Este significado le otorga un papel en la enseñanza que no requiere justificaciones adicionales. Un ejemplo de este uso del término puede encontrarse en Artigue (1992).

En otras ocasiones, se considera que el significado de epistemología está asociado con lo que podemos nombrar, con menos ambigüedad, como filosofía de la ciencia, disciplina en la cual el interés está centrado en el análisis de los supuestos de las teorías científicas o de las formas de validación de las mismas antes que en las circunstancias psicológicas o sociológicas que permiten su producción ${ }^{4}$. Es esta última acepción la que voy a considerar en lo que sigue. Tomado el término con este segundo significado, hay una convicción generalizada acerca de la importancia de la epistemología en la enseñanza de las ciencias. Uno de los objetivos de este trabajo será mostrar ciertos tipos de casos en los que se manifiesta su relevancia. El otro será identificar un rol para los filósofos de la ciencia dentro de los grupos de investigación en enseñanza.

\section{ENTRE LA TEORÍA ENSEÑADA Y LA TEO- RÍA DE LOS CIENTIFICOS}

Chevallard (1991) introdujo el término transposición didáctica para nombrar el proceso de transición que va del «objeto de saber» al «objeto de enseñanza». Si bien su objetivo estaba centrado en la didáctica de la matemática, su uso se extendió a la didáctica de otras disciplinas científicas. Es indudable que tal concepto permite dar cuenta de una realidad de la enseñanza que todo docente conoce: la teoría de los científicos y la teoría enseñada por el profesor no son idénticas, como tampoco lo es la aprendida por los alumnos. En buena medida esto es producto de las necesarias adaptaciones que debe hacer el docente para lograr algún grado de aprendizaje por parte de los alumnos. Esto lo lleva a adaptar el lenguaje, el tipo de herramientas matemáticas que utiliza, el grado de simplificación de las aplicaciones, la idealización de los ejemplos, etc.

Este proceso que establece una diferencia entre ambas teorías (la de los científicos y la enseñada) conduce a una diferencia mayor entre ambas cuanto menor es el nivel educativo sobre el que se trabaja. Es decir, la distancia entre ambas será mucho mayor en el nivel inicial de educación que lo que lo será en el último ciclo de la educación general básica ${ }^{5}$. Si la enseñanza, además de sus desiderata formativos, persigue también enseñar contenidos, es decir, teorías científicas en el caso que nos ocupa, entonces es de esperar que la teoría de los científicos y la teoría enseñada tiendan a parecerse cada vez más a medida que se avanza en los niveles educativos. Para tratar de dar una imagen gráfica podríamos pensar la teoría de los científicos como una recta a la cual asintóticamente se acerca (sin cortarla) una curva que representa la teoría enseñada a medida que el nivel educativo crece.

Para analizar si esto es así, deberíamos hacer un seguimiento diacrónico de la enseñanza de una teoría o algún entramado conceptual. Por ejemplo, podría estudiarse cómo se enseña la geometría euclidiana en los distintos niveles de enseñanza y ver si dicha convergencia se manifiesta llegando a los niveles de la enseñanza universitaria. Lo mismo podría hacerse de la teoría de Newton, para tomar sólo dos ejemplos de contenidos insoslayables. El trabajo en sí consistirá en analizar la teoría de los científicos y luego ver qué se enseña en cada nivel, observando desde la actividad del profesor en el aula hasta la bibliografía que utiliza para tal fin.

Uno de los puntos de interés de este trabajo se centra en mostrar cómo en la transposición didáctica se dejan de lado ciertas cuestiones relacionadas con lo que más arriba nombré como filosofía de la ciencia, lo que impide, entre otras cosas, la convergencia mencionada, incluso en el nivel de las carreras universitarias específicas ${ }^{6}$.

En lo que sigue voy a presentar una serie de citas extraídas de manuales de uso habitual en la enseñanza de las ciencias $^{7}$, tanto de nivel medio como universitario, en las que se detecta cómo la falta de un correcto análisis epistemológico conduce a:

a) circularidades o contradicciones que generan confusión en los alumnos;

b) confusiones que pueden conducir a una mala comprensión de la metodología de la ciencia;

c) la enseñanza de teorías diferentes de las desarrolladas por los científicos, sin que se logre la mencionada convergencia. 
Estos casos permitirán ver que la filosofía de la ciencia tiene, además de un rol formativo en los docentes de ciencia, un rol que cumplir en la crítica de las estrategias actuales (detectando errores, confusiones y supuestos) y, por ende, en el desarrollo de nuevas estrategias (construidas sobre la base de las críticas). Esto justifica no sólo la necesidad de que los docentes de ciencia se formen en filosofía de la ciencia sino también la integración de filósofos de la ciencia, y no tan sólo la de investigadores con formación en filosofía de la ciencia en grupos de investigación interdisciplinarios que persiguen el mejoramiento de la enseñanza de las ciencias.

\section{LA DEFINICIÓN DE MAGNITUD FÍSICA Y EL JUEGO DE LA ESCONDIDA}

Veamos un caso de lo que puede encontrarse en los libros de texto de enseñanza de física. Podemos leer:

«Cada proceso de medición define lo que se llama una magnitud física. Estas quedan unívocamente determinadas por el proceso de medición [...] Hay muchos procesos de medición que definen una misma magnitud [...] Son procesos de medición equivalentes.» (Roederer, 1979).

Es posible encontrar una circularidad en la cita anterior. La definición dada anteriormente estipula a qué se llama magnitud física. Una definición de este tipo es una equivalencia entre lo definido y lo que se busca definir. En nuestro caso, proceso de medición y magnitud física son equivalentes. Si se encontrasen procesos de medición que definiesen una misma magnitud, entonces dichos procesos serían equivalentes, pues las magnitudes son equivalentes, justamente por ser la misma. Ahora bien, para saber si es la misma magnitud, debemos chequear si se utiliza el mismo proceso para medirlas o bien si se utilizan procesos equivalentes, pues esto es lo que se requiere para que las magnitudes así definidas resulten equivalentes.

En definitiva, lo que el autor nos dice puede expresarse diciendo:

«Son procesos equivalentes aquellos que definen la misma magnitud y se tiene la misma magnitud si está definida por procesos equivalentes.»

Esta circularidad proviene del intento de evitar proponer entidades físicas que no estén definidas por una operación de medición. Sabemos ya que este operacionalismo extremo no permite resolver el problema de disponer de distintos mecanismos de medición para la misma magnitud sin caer en una proliferación absurda de entidades con las mismas propiedades. Tendríamos, por ejemplo, una magnitud longitud medida por comparación con un patrón, otra longitud 2 medida por reflexión de rayos de luz, etc.

Existen, por supuesto, respuestas alternativas a este tipo de problemas. Como ejemplo, tomemos el caso de la mecánica de Newton y, en particular, la magnitud «fuerza». $\mathrm{Al}$ igual que lo mencionado para la longitud, tendríamos una fuerza peso definida por la balanza de platillos, otra medida mediante un dinamómetro, etc. En este caso, el problema quedaría resuelto si se considerase que la fuerza es una magnitud física que no es definible dentro de la teoría, sino que se admite como primitivo ${ }^{8}$ y que puede ser medida por un conjunto diferente de métodos. Claro que esto tiene supuestos que buena parte de los físicos se resisten a admitir ${ }^{9}$. Sin embargo más interesante que la respuesta que se puede brindar frente a este problema, lo que importa, a los fines de lo que se desea mostrar en este trabajo, es que en la enseñanza será necesario llevar a cabo una discusión que involucre las diversas alternativas. Esta discusión debería incluir la viabilidad del operacionalismo ${ }^{10} \mathrm{O}$ los supuestos que tiene la física e, incluso, la ciencia en general, junto con una toma de conciencia sobre los compromisos que trae aparejado el adoptar una u otra posición respecto del problema.

Podría también interpretarse que en la cita anterior no hay una circularidad. Para entenderlo de esta manera, sin embargo, sería necesario admitir que definir, en la cita anterior, no significa establecer una equivalencia. Esta interpretación creo que es un tanto forzada pero, de todas formas, si es la que supone el autor del texto, nuevamente justifica la discusión referida en el párrafo anterior.

Este ejemplo muestra una falta de análisis filosófico. Es posible que este análisis no sea requerido por los científicos en su trabajo cotidiano pero, en lo que a la enseñanza se refiere, debe realizarse para no introducir errores epistemológicos, ya sea en la bibliografía o en el marco mismo del aula. Uno de los problemas que suscita este ejemplo es la posible inducción en los alumnos de una contradicción en su formación física. La contradicción es consecuencia de no distinguir entre operacionalización de una magnitud, es decir, definir un mecanismo operativo para detectar la presencia de la magnitud o medir una cantidad de ella; y operacionalismo, es decir, definir la magnitud por la operación anterior. Un correcto análisis epistemológico del tema permitirá el desarrollo de una nueva vía de enseñanza de qué es y cómo se detecta una magnitud física.

\section{LA GEOMETRÍA O VARIOS GATOS EN LA MISMA BOLSA}

La geometría euclidiana se enseña en la escuela primaria y en el nivel medio. Una manera habitual de enseñarla es presentar axiomas y luego deducir teoremas. Simultáneamente con esto se realizan, en clase, experiencias de comprobación experimental de las propiedades deducidas. Por ejemplo, una vez presentada la axiomática de la geometría y definidos los triángulos, se demuestra que la suma de sus ángulos interiores es $180^{\circ}$. Una vez hecho esto se decide construir un triángulo (ya sea recortándolo en cartulina o dibujándolo en un papel) y medir sus ángulos interiores con el fin de comprobar que efectivamente la suma da $180^{\text {ol1 }}$.

Las actividades de este tipo son realizadas en la mayor parte de las ocasiones sin que se cuestione el rol que cumplen la demostración y la comprobación experimental. Lo 
que subyace en este ejemplo es la distinción entre geometría matemática y geometría física, que suele no ser clara en ciertos niveles (sobre todo en primario y medio). Si se está desarrollando la geometría matemática, ¿qué sentido tiene la comprobación experimental? Y si se está desarrollando la geometría física, ¿qué permite concluir la comprobación experimental?, ¿qué significa la demostración del teorema en este caso?

Otro ejemplo del mismo problema: un libro de texto secundario de matemática, muy difundido entre los docentes de la materia (Tapia, 1987), presenta los axiomas de la geometría de manera clásica:

Axioma 1: Existen infinitos puntos.

Axioma 2: Una recta es un conjunto de infinitos puntos, etc.

A continuación ofrece a los estudiantes una serie de ejercicios, entre los que encontramos:

«Diego pretende apoyar un trozo de cartón sobre las puntas de dos lápices. ¿Crees que se mantendrá fijo? ¿Cuál es el menor número de lápices que se necesita para que el cartón quede fijo?»

Y a continuación:

«Demuestra que si $\mathrm{R} \wp \alpha$, existe por lo menos una recta de $\alpha$ que corta a R.»

...teniendo en cuenta que previamente se indicó que las letras griegas nombran planos y las mayúsculas, rectas.

Claramente se ve que el primer ejercicio conduce a la comprobación de que la geometría física responde a la axiomática euclidiana y, por lo tanto, la validación del conocimiento adquirido, en este caso, tiene bases experimentales, es decir, es el de las ciencias fácticas. En el segundo caso, la pretensión es deducir la afirmación a partir de los axiomas y así la validación del conocimiento es el de las ciencias formales.

Nuevamente nos podemos preguntar si los estudiantes pueden saber que están aprendiendo simultáneamente geometría matemática y geometría física y distinguir las distintas formas de, por ejemplo, validar afirmaciones en un caso y otro sin que se establezca una discusión al respecto ${ }^{12}$. En relación con este punto vale la pena aclarar que no estoy proponiendo que la enseñanza de la geometría deba realizarse desde una sola perspectiva ni que pretendamos en los primeros niveles someter a los alumnos a este tipo de discusiones, pero sí que se tenga en cuenta que, a partir del nivel medio, la discusión debe proponerse en algún momento. Ya sea que se enseñe geometría desde un punto de vista fáctico, es decir, como geometría física, o desde el formal (en cualquier caso posteriormente deberá enseñarse la otra), la distinción entre uno y otro conocimiento debe también llevarse a cabo ${ }^{13}$.

Uno de los objetivos de la enseñanza es introducir a los estudiantes en la metodología de trabajo de la disciplina que se trata. El ejemplo recién expuesto puede llevar a los alumnos a asociar metodológicamente físi- ca y matemática o ciencias fácticas y ciencias formales ${ }^{14}$.

\section{EL PRINCIPIO DE MASA O CÓMO CAMBIAN LOS SUPUESTOS}

Consideremos otro caso. La física de Newton tiene entre sus principios la ya muy conocida ley de masa, según la cual la fuerza neta y la aceleración que soporta un cuerpo son directamente proporcionales, con una constante de proporcionalidad que depende de la masa del cuerpo. Habitualmente es expresada en lenguaje matemático como: $F=m a^{15}$.

Suele presentarse la dinámica de Newton tanto en la escuela media como en los primeros cursos universitarios (incluyendo aquí los de formación de los futuros profesores) y partiendo de los conocidos tres principios: inercia, masa e interacción. Cuando se presenta el segundo, pueden encontrarse en los textos expresiones como:

«Cuando varias fuerzas obran sobre un cuerpo, cada una produce su propia aceleración independientemente» (Resnick, 1973).

O bien:

«La fuerza que actúa sobre un cuerpo es igual al producto de la masa del cuerpo por la aceleración que le imprime.» (Alonso, 1979).

Otro caso lo podemos leer en un interesante libro de física (Hewitt, 1995), muy apreciado por su nivel conceptual. En la presentación de la segunda ley de Newton dice, luego de recordar la definición de aceleración:

«En este capítulo nos ocuparemos de la causa de la aceleración, o sea, de la fuerza.» ${ }^{16}$

Mas adelante formula la segunda ley como sigue:

«La aceleración que adquiere un objeto por efecto de una fuerza total es directamente proporcional a la magnitud de la fuerza total, tiene la misma dirección que la fuerza total y es inversamente proporcional a la masa del cuerpo considerado.»

Lo interesante de las citas es que su lenguaje esconde una relación causal: es la fuerza la que produce, en un caso, e imprime la aceleración, en otro, para finalmente ser la causa de la aceleración. Estas citas no son diferentes de las que pueden encontrarse en cualquier otro libro de texto de física ni tampoco de cómo se presenta el principio de masa en los cursos, tanto en el nivel medio como en el universitario. En general en la presentación de este principio se utiliza un lenguaje causal que supone que la fuerza es causa de la aceleración, aunque la teoría no presuponga causalidad alguna entre fuerza y aceleración ${ }^{17}$. Obsérvese que en la formulación de Hewitt (la última cita) puede reemplazarse la referencia causal que se realiza al utilizar el término efecto sin que cambie el sentido de la ley:

«La aceleración que adquiere un objeto es directamente proporcional a la magnitud de la fuerza total aplicada so- 
bre el mismo, tiene la misma dirección que la fuerza total $\mathrm{y}$ es inversamente proporcional a la masa del cuerpo considerado.»

Lo que la ley expresa es simplemente que dos magnitudes, fuerza y aceleración, son proporcionales; no se menciona ninguna relación asimétrica que pueda confundirse con causalidad. Una característica básica de la relación de causalidad es que entre el evento causa (C) y el evento efecto (E) se establece una relación asimétrica que justifica que pueda afirmarse que $\mathrm{C}$ es causa de $\mathrm{E}$ pero no que $\mathrm{E}$ es causa de C. En el caso de fuerza y aceleración, ni siquiera hay diferencia temporal entre ambas, ya que ambas aparecen simultáneamente. Es decir que ejercer una fuerza o ejercer una aceleración es equivalente en la práctica, aunque se usen distintas expresiones cuando se intenta focalizar el interés en una de ellas en particular pero por razones extrateóricas.

Tal vez puedan encontrarse buenas razones para el uso del lenguaje causal en este caso. Por ejemplo, si se intenta experimentar, nos parece más sencillo ejercer una fuerza sobre un cuerpo que aplicar una aceleración sobre el mismo. Pero entiendo que esto es por la asociación que establecemos entre el significado de fuerza en el lenguaje cotidiano y aquel del lenguaje de la física de Newton. Es posible que la asociación con la causalidad provenga de lo que parece más sencillo de manipular. Parece más fácil manipular fuerzas que aceleraciones (aunque podría argumentarse que esto es sólo una manera de hablar). Podrían también encontrarse razones históricas para este modo de enseñanza (quienes hoy enseñan aprendieron con este lenguaje y es probable que no se les haya planteado nunca la discusión).

Aunque no resulte habitual (y tal vez tampoco útil) se podría enunciar el principio de masa diciendo:

«[...] si se ejerce una aceleración $a$ sobre un cuerpo de masa $m$ aparece sobre el cuerpo una fuerza $F$ cuya intensidad, dirección y sentido viene dada por $F=m a »$.

...que esconde un lenguaje causal pero invirtiendo la relación habitual. Aquí la formulación da idea de que la aceleración es causa de la fuerza. En la visión del mundo mecánico nada cambia si se toma, ya sea la formulación no causalista (la de Hewitt corregida que mencioné arriba) o la causalista, que invierte el sentido habitual de la relación, como en el último caso, en reemplazo de la tradicional.

No pretendo agotar acá la discusión acerca de la segunda ley de Newton y la presuposición o no de la relación causal. Lo que resulta interesante y que muestran las citas anteriores es que los presupuestos teóricos no necesariamente son los mismos que los que se vuelcan al enseñar. El principio de masa no supone relación causal alguna y, sin embargo, en su enseñanza se presenta habitualmente como si la fuerza fuera causa de la aceleración.

Pero aun en el caso en que se siga dudando sobre si está presente o no la causalidad en el principio de masa, el problema que nos ocupa se mantiene. La presentación del principio se hace suponiendo la causalidad pero no se presenta la alternativa no causal para someterla a discusión ${ }^{18}$.
Un punto interesante que muestra este ejemplo es que entre la teoría desarrollada por los científicos y la que enseña el profesor existen, además de las simplificaciones y adaptaciones involucradas en la trasposición didáctica que suelen reconocerse explícitamente, otras diferencias: en los supuestos, en el tipo de ordenamiento del mundo que presuponen, en el tipo de relaciones, etc. La convergencia referida más arriba no se verifica en este aspecto aunque se avance en el nivel educativo.

\section{FILOSOFÍA DE LA CIENCIA Y ENSEÑANZA DE LAS CIENCIAS}

Los ejemplos expuestos arriba muestran la importancia que la filosofía de la ciencia tiene en la formación del enseñante, sea éste del nivel educativo que sea. Existen dos objetivos cuyo logro justifica la necesidad de una formación epistemológica (siempre considerando el término en el segundo de los sentidos dados en la introducción) de los docentes de ciencia:

1) Poder sostener con sus alumnos discusiones con un nivel razonable de profundidad en ciertos temas, tales como el planteado en el ejemplo de la definición operacional de magnitud física.

2) Poder analizar los supuestos que está asumiendo en sus clases o en sus textos cuando enseña alguna teoría y también los supuestos epistemológicos de los currículos escolares.

El segundo es importante, pues permite que el docente se prevenga de inducir problemas de aprendizaje; o dicho en términos con reminiscencias bachelardianas ${ }^{19}$, evitar inducir obstáculos epistemológicos que luego impidan las rupturas necesarias para el aprendizaje.

En segundo lugar, de los ejemplos surge que la filosofía de la ciencia tiene un rol que cumplir en la investigación en la enseñanza de las ciencias. Por citar solo un caso, el hecho de sacar a la luz los supuestos de una disciplina tal como la desarrollan los científicos y de esta forma analizar qué dificultades pueden inducirse en los estudiantes utilizando una determinada técnica de enseñanza.

Un grupo interdisciplinario de investigación en enseñanza de las ciencias no puede prescindir de los filósofos. Tienen, los filósofos de la ciencia, una misión específica (aunque no la única) dentro de estos grupos: detectar aquellos supuestos (en general no científicos) que se deslizan en el proceso de trasposición y que son diferentes en la ciencia de los científicos y en la ciencia enseñada en las aulas en los distintos niveles. El punto básico de esta tarea está en la investigación e identificación, no en la normativa. La decisión de qué conceptos, supuestos, etc. vale la pena sacrificar (o es conveniente hacerlo) en determinado nivel de la enseñanza para recuperarlo en otro será decisión del grupo interdisciplinario (y desde ahora motivo de investigación posterior sobre la decisión tomada), pero se habrá evitado que se generen problemas por el simple desconocimiento. 
Debo dejar en claro que no me refiero a los supuestos de la enseñanza de las ciencias en tanto disciplina científica, sino a los supuestos que se tienen acerca de la ciencia o de alguna teoría científica particular al reproducirla en la enseñanza. La discusión epistemológica sobre algunos temas, el estatus de la enseñanza de las ciencias como disciplina científica, la forma de validar afirmaciones, etc. no deja de ser interesante pero no es el objeto de este trabajo.

Intentaré hacer una comparación, aunque tal vez no resulte del todo feliz, como suele ocurrir con las comparaciones. En otro ámbito de la filosofía, el de la ética, se desarrolló una rama, subsidiaria de la anterior, pero bien diferenciada: la ética aplicada. En buena medida, el surgimiento de esta subrama estuvo motivada por las demandas de la

\section{NOTAS}

* Este trabajo fue realizado en el marco del proyecto de investigación «Leyes, causa, condicionales y cambio de teoría», que es financiado por UBACyT y dirigido por Eduardo H. Flichman, a quien agradezco junto a Hernán Miguel y Horacio Abeledo los comentarios realizados a las versiones previas. Doy las gracias también al responsable de esta revista por sus útiles sugerencias.

${ }^{1}$ Como ejemplos pueden verse Matthews (1994), Adúriz-Bravo, Izquierdo (2001), Gil Pérez (1993), Duschl, Gitomer (1991) o la serie de trabajos compilados en McComas (1998).

${ }^{2}$ Uno de los pocos que lo hicieron fue Kuhn, aunque debería tenerse en cuenta que dicho autor es, además de filósofo e historiador de la ciencia, físico, cosa que probablemente haya influido para despertar su interés en estos temas.

${ }^{3}$ En cambio es bastante habitual encontrar trabajos que apliquen otra rama de la filosofía, la ética, a situaciones concretas, muchas de ellas vinculadas con casos médicos.

${ }^{4}$ Sigo aquí, con leves diferencias, la caracterización que hace Klimovsky (1994).

${ }^{5}$ En la Argentina la enseñanza obligatoria abarca un nivel inicial en el que ingresan alumnos con cinco años de edad y el nivel de educación general básica (EGB), que está dividido en tres ciclos cada uno de los cuales abarca tres años. El siguiente nivel abarca tres años de enseñanza polimodal con estudiantes que tienen entre 15 y 18 años de edad.

${ }^{6}$ Es claro que la convergencia de la que hablé arriba debería ser mayor, por ejemplo, en el caso del físico que en el del ingeniero si lo que se está enseñando es la teoría física.

${ }^{7}$ Me refiero a lo que ocurre en la Argentina, aunque no creo que la situación sea diferente en otros países.

${ }^{8}$ Un término primitivo es aquél que no se define y a partir del cual se definen los restantes términos del lenguaje. En el caso de la mecánica de Newton puede considerarse fuerza como primitivo o bien puede también definirse a partir de otros como por medicina y la biología que apelan periódicamente a los filósofos dedicados a la ética para que se integren en sus grupos a fin de discutir situaciones éticas particulares, que pueden tener que ver, por ejemplo, con la corrección o no desde el punto de vista ético de la realización de cierta experiencia o con la toma de decisión en casos médicos complejos. De la misma forma entiendo que, a partir de las necesidades de la enseñanza y de la investigación en enseñanza, comienza a desarrollarse lo que podríamos llamar filosofía de la ciencia aplicada, una subrama de la filosofía de la ciencia con tareas específicas: análisis de los aspectos epistemológicos que quedan a un lado o son modificados en la trasposición, recomendaciones acerca de aquéllos que sería útil analizar en cada nivel de enseñanza, etc. ejemplo «cantidad de movimiento» 0 «impulso lineal». Para una introducción en la discusión de esta problemática puede verse Hempel (1983) y Flichman, Miguel, Paruelo y Pissinis (1999).

${ }^{9}$ Para lograr esto deberían abandonar su pretensión operacionalista y admitir que en su trabajo científico asumen supuestos metafísicos de los cuales no pueden apartarse. Dificultades similares se presentan entre los profesores de física, con el consecuente traslado al aula de esa dificultad.

10 «Operacionalismo» es el nombre con el que se conoce la corriente inaugurada por Bridgman (1927) y que sostiene lo descrito. Una discusión más detallada sobre sus dificultades puede encontrarse en Hempel (1983).

${ }^{11}$ Agradezco a Hernán Miguel el recuerdo de esta actividad que realizábamos en nuestras escuelas en lo que ahora sería el tercer ciclo de la EGB.

${ }^{12}$ De la misma manera nos podemos preguntar si los docentes, sobre todo en los primeros niveles, tienen clara la diferencia, dado que la discusión en los institutos de formación adolece de las mismos problemas que se mencionan en este trabajo.

${ }^{13}$ Otro punto interesante de esto es que permite integrar los conocimientos obtenidos en las distintas disciplinas enseñadas en la escuela. La experiencia docente muestra que los alumnos que ingresan en la universidad no consiguen vincular, por ejemplo, matemática y física. Una discusión como la propuesta permitiría establecer dichos vínculos.

${ }^{14}$ Respecto de este punto tengo la convicción de que la enseñanza desde lo concreto a lo abstracto, en tanto se pueda, es más efectiva que el acceso desde lo abstracto. En este sentido creo que la enseñanza de la geometría en particular debería comenzar por la geometría física y, luego de que los conceptos han sido incorporados convenientemente (se han asimilado como para tener «intuiciones» del objeto de estudio), pasar a la enseñanza de la geometría matemática. 
${ }^{15}$ Se indican en cursiva las magnitudes vectoriales y, en formato normal, las escalares.

${ }^{16}$ Las cursivas son del autor.

${ }^{17}$ En Miguel y Paruelo (1997) se sostiene que la relación de causación no es una genuina relación entre eventos sino que es una proyección que el hablante efectúa sobre el mundo. En el caso de la ley de Newton, cuando se sostiene que la ley no presupone la relación causal entre fuerza y aceleración, se hace independientemente de la postura mencionada respecto de dicha relación. Alguien que sostenga que existen relaciones causales admitiría de todas formas que, en este caso, tal relación no está

\section{REFERENCIAS BIBLIOGRÁFICAS}

ADÚRIZ-BRAVO, A. y IZQUIERDO, M. (2001). The philosophy of science in prospective science teacher education. Rationale and practical proposals. Publicación electrónica de trabajos presentados en la 26th Annual Conference Stockholm 2001 de la Association for Teacher Education in Europe. http:// www.lhs.se/atee/papers/RDC_2_the_philosophy.doc

ARTIGUE, M. (1992). The Importance and limits of Epistemological Work in Didactics, en Geesling, W., Graham, K. (eds.). Proceedings of the $16^{\text {th }}$ PME Conference, V3, University of New Hampshire, Durham.

ALONSO, M. y ROJO, O. (1979). Física (Mecánica y Termodinámica). México: Fondo Educativo Interamericano.

BACHELARD, G. (1993). La formación del espíritu científico. (1a. ed., 1938). México: Siglo XXI Editores.

BRIDGMAN, P. (1927). The Logic of Modern Physics. Nueva York: The Macmillan Company.

CHEVALLARD, Y. (1991). La transposición didáctica. Del saber sabio al saber enseñado. Buenos Aires: Aique.

DUSCHL, R.A. y GITOMER, D.H. (1991). Epistemological perspectives on conceptual change: implications for educational practice. Journal of Research in Science Teaching, 28(9), pp. 839-858.

FLICHMAN, E., MIGUEL, H., PARUELO, J. y PISSINIS, G. (eds.) (1999). Las raíces y los frutos. Buenos Aires: CCC Educando.

GIL PÉREZ, D. (1993). Contribuciones de la historia y la filosofía de las ciencias al desarrollo de un modelo de enseñanza- presente, en virtud de la asimetría faltante que menciono más adelante.

${ }^{18}$ No estoy con esto proponiendo que haya que eliminar el lenguaje causal en la enseñanza del segundo principio. Por el contrario, creo que este lenguaje, para empezar la enseñanza, es correcto. Lo que sí sostengo es que, si se usa un lenguaje causal, debe en alguna etapa del proceso de enseñanza hacerse explícito que la ley no presupone la relación de causalidad, o al menos plantear la discusión.

${ }^{19}$ Hago referencia al concepto de obstáculo epistemológico presentado por Bachelard (1993). aprendizaje como investigación. Enseñanza de las Ciencias, 11(2), pp. 197-212.

HEMPEL, C. (1983). Filosofía de la ciencia natural. Madrid: Alianza Editorial.

HEWITT, P.G. (1995). Física conceptual. Addison-Wesley Iberoamericana.

KLIMOVSKY, G. (1994). Las desventuras del conocimiento científico. Buenos Aires: A-Z Editora.

MATTHEWS, M.R. (1994). Historia, filosofía y enseñanza de las ciencias: la aproximación actual. Enseñanza de las Ciencias, 12(2), pp. 255-277.

McCOMAS, W. (ed.) (1998). The nature of science in science education: Rationales and strategies. Dordrecht: Kluwer Academic Publishers.

MIGUEL, H. y PARUELO, J. (1997). Causación, producción y función. Crítica, Revista Hispanomericana de Filosofía, XXIX, 87, pp. 53-90. México.

RIESNICK, R. y HALLIDAY, D. (1973). Física. México: CECSA.

ROEDERER, J. (1979). Mecánica Elemental. Buenos Aires: EUDEBA. (1a. ed., 1963. Existen reediciones posteriores a 1979.)

TAPIA, N.V. DE, TAPIA, A. y TAPIA, C.A.: Matemática 1. (Editado desde 1987 y reeditado anualmente hasta la fecha). Buenos Aires: Editorial Estrada. 